UBCTP93-010; July 1993

\title{
SCATTERING FROM A TWO DIMENSIONAL ARRAY OF FLUX TUBES: \\ A STUDY OF THE VALIDITY OF MEAN FIELD THEORY
}

\author{
Ken Kiers and Nathan Weiss \\ Department of Physics, University of British Columbia, \\ Vancouver, B.C. V6T 2A6, Canada
}

\begin{abstract}
Mean Field Theory has been extensively used in the study of systems of anyons in two spatial dimensions. In this paper we study the physical grounds for the validity of this approximation by considering the Quantum Mechanical scattering of a charged particle from a two dimensional array of magnetic flux tubes. The flux tubes are arranged on a regular lattice which is infinitely long in the " $y$ " direction but which has a (small) finite number of columns in the " $x$ " direction. Their physical size is assumed to be infinitesimally small. We develop a method for computing the scattering angle as well as the reflection and transmission coefficients to lowest order in the Aharonov-Bohm interaction. The results of our calculation are compared to the scattering of the same particle from a region of constant magnetic field whose magnitude is equal to the mean field of all the flux tubes. For an incident plane wave, the Mean Field approximation is shown to be valid provided the flux in each tube is much less than a single flux quantum. This is precisely the regime in which Mean Field Theory for anyons is expected to be valid. When the flux per tube becomes of order 1, Mean Field Theory is no longer valid.
\end{abstract}

Typeset Using REVTEX 


\section{INTRODUCTION}

There has been much interest in recent years in the phenomenon of fractional statistics in two spatial dimensions. Particles possessing fractional statistics, known as anyons, besides being interesting in their own right, have found applications in the Quantum Hall Effect [1] and have also been investigated in the context of High $T_{c}$ Superconductivity.

Anyons are often studied in the framework of "Chern-Simons" theory in which they are modeled by "attaching" an infinitesimal tube of "statistical" flux to each particle in the system. In order to calculate various properties of the anyon gas it is often assumed that each anyon "feels" as if it were traveling in the mean magnetic field due to all of the other anyons in the system. This "Mean Field Theory" has been quite successful at describing many properties of anyon systems including Anyon Superconductivity [2] and the Fractional Quantum Hall Effect [3]. In fact in Chern-Simons Field Theory, the mean field approximation can be shown to be valid when the statistical flux per particle is a small fraction of a flux quantum. Classically this Mean Field (MF) idea seems absurd since the magnetic field is zero except at isolated singularities and so there is no Lorentz force. Quantum mechanically however the vector potentials themselves attain importance [4. Thus it seems at least plausible that a particle moving in a region populated by flux tubes would in some respects behave as though it were in a mean magnetic field. It is this idea which we investigate in this paper.

It is quite straightforward to see that Mean Field Theory is at best valid only when the flux per particle is not too large. Assume that a given anyon "feels" a mean field and hence travels in a circular (Landau) orbit. If the area enclosed by the orbit contains, on average, many other anyons then the approximation may be valid. Proceeding in this way one finds both for models based on bosons [5] and on fermions [6] that the MF approximation is self-consistent only when the flux per particle is much less than a single flux quantum.

It is our goal in this paper to examine the physical ideas implicit in the above argument in more detail. One question of interest in this context is: To what extent does a particle traveling in the presence of a number of infinitesimally small flux tubes really behave as though it were in some mean magnetic field? In other words, to what extent does the presence of the gauge field mimic the effect of a spatially constant statistical magnetic field? Ideally we could consider an array of infinitesimally small flux tubes and study the quantum mechanical motion of either a charged particle or another flux tube in the presence of this array. This however turns out to be a very difficult problem to solve [7]. We choose instead to consider a specific configuration of flux tubes consisting of a lattice which is of infinite extent in the $y$ direction but which contains a (small) finite number of columns in the $x$ direction. A related problem (in which the array is random) has been considered previously in Ref. [8] in which scattering from the array was considered as an incoherent sum of the scattering off each flux tube. In this paper we consider the coherent scattering of a charged particle incident on a lattice of flux tubes. The method which we develop is a variation on first order scattering theory. Our main objective will be to compute the scattering angle and to compare the result with scattering from a strip of constant magnetic field.

We thus begin in Section II by calculating the scattering angle of a plane wave incident on an infinitely long strip of constant magnetic field. In Section III, after calculating some analytic results, we develop a formalism, similar in spirit to the approach conventionally 
used to compute the index of refraction from the microscopic scattering amplitude [9, 10], to study scattering of a charged particle from a lattice of flux tubes. We then use this formalism to find an expression for the scattering angle to first order in the interaction, which we then compare to the analytic results. (Note that while exact solutions are available for scattering from one [4] and, more recently, two [11] flux tubes, the problem of scattering from an infinite array of tubes cannot be completely solved analytically. We will find however that it is possible to solve for the scattering angle exactly in a limited number of cases.) In Sections II and III we also discuss the reflection and transmission amplitudes. Section IV contains a discussion of the results.

\section{SCATTERING FROM A STRIP OF CONSTANT MAGNETIC FIELD}

In this section we briefly review the result of scattering a charged particle from an infinitely long magnetic strip in two spatial dimensions. We thus consider a situation in which the magnetic field $B$ is constant in the region $0 \leq x \leq d$. It is convenient to work in a gauge in which $A_{x}=0$ and

$$
A_{y}= \begin{cases}-B d / 2, & \text { if } x \leq 0 \\ B x-B d / 2, & \text { if } 0 \leq x \leq d \\ B d / 2, & \text { if } x \geq d\end{cases}
$$

The Schrödinger equation for a particle with charge $e$ subject to the above magnetic field is given by

$$
-\frac{1}{2 m}[\nabla-i e \mathbf{A}]^{2} \psi(x, y)=E \psi(x, y) .
$$

This equation is solved by separating the $x$ and $y$ dependence and representing the latter as a plane wave. We thus write

$$
\psi(x, y)=e^{i\left(k_{y}+\frac{e B d}{2}\right) y} f(x)
$$

with

$$
\frac{1}{2 m}\left[-\frac{d^{2}}{d x^{2}}+\left(k_{y}+\frac{e B d}{2}-A_{y}\right)^{2}\right] f(x)=E f(x)
$$

Suppose that the scattering particle is incident from the right (positive $x$ ). The incident wave

$$
\psi_{\text {inc }}=e^{i\left(k_{y}+\frac{e B d}{2}\right) y} e^{-i k_{x} x}
$$

will solve the Schrödinger equation for $x>d$ provided $2 m E=k_{x}^{2}+k_{y}^{2}$.

In the interior region $(0 \leq x \leq d)$ the particle behaves as if it were in a potential which is a truncated parabola. Although an explicit solution is in general not available, it is rather straightforward to obtain a series solution. For certain combinations of the parameters the 
series truncates and the wave function is simply a Hermite polynomial but in general the series is infinite. (In the case when the series truncates, the wave function is related to that of the Landau level.) For $x<0$ the wave function is again given by a plane wave

$$
f(x)=e^{-i \tilde{k}_{x} x}
$$

but now

$$
2 m E=\tilde{k}_{x}^{2}+\left(k_{y}+e B d\right)^{2}
$$

It thus follows that for $x<0$

$$
\tilde{k}_{x}=\sqrt{k_{x}^{2}-e^{2} B^{2} d^{2}-2 k_{y} e B d}
$$

Transmission of the particle to the region $x<0$ can occur if the energy is sufficiently large so that $k_{x}^{2} \geq e^{2} B^{2} d^{2}+2 k_{y} e B d$. In this case the solution to Eq. (2) can be written as

$$
\psi=e^{i\left(k_{y}+\frac{e B d}{2}\right) y} \times \begin{cases}\tau e^{-i \tilde{k}_{x} x} & x \leq 0 \\ e^{-\mathrm{X}^{2} / 2}\left(c_{0} G_{0}(\mathrm{X})+c_{1} G_{1}(\mathrm{X})\right) & 0 \leq x \leq d \\ \left(e^{-i k_{x} x}+\rho e^{i k_{x} x}\right) & x \geq d\end{cases}
$$

where $\tilde{k}_{x}$ is given by Eq. (8) and

$$
\mathrm{X}=\sqrt{|e B|}\left(x-\left(\frac{k_{y}}{e B}+d\right)\right)
$$

is proportional to the distance from the centre of the parabola. The functions $G_{0}$ and $G_{1}$ are given by

$$
\begin{gathered}
G_{0}(\mathrm{X})=1+\frac{2(-\lambda)}{2 !} \mathrm{X}^{2}+\frac{2^{2}(2-\lambda)(-\lambda)}{4 !} \mathrm{X}^{4}+\cdots \\
G_{1}(\mathrm{X})=\mathrm{X}+\frac{2(1-\lambda)}{3 !} \mathrm{X}^{3}+\frac{2^{2}(3-\lambda)(1-\lambda)}{5 !} \mathrm{X}^{5}+\cdots \\
\text { with } \quad \lambda=\frac{1}{2}\left(\frac{k_{x}^{2}+k_{y}^{2}}{|e B|}-1\right)
\end{gathered}
$$

$c_{0}, c_{1}, \rho$, and $\tau$ are constants which are fixed by requiring that the wave function and its derivative be continuous across the two boundaries. $\rho$ and $\tau$ are the reflection and transmission amplitudes respectively. Note that the series (11) and (12) converge for all $\mathrm{X}$. (To see this note that for the high order terms in the expansion the ratio of successive terms behaves like the corresponding ratio in the $\operatorname{expansion}$ of $\exp \left(2 \mathrm{X}^{2}\right)$ [12].) If the energy is small so that $k_{x}^{2}<e^{2} B^{2} d^{2}+2 k_{y} e B d$ there is no transmission and one obtains instead a decaying exponential for $x<0$ so that the wave is totally reflected. 
It is quite straightforward to compute the angle by which the particle is scattered. The simplest way to do this is to compute the gauge invariant current density given by

$$
\mathbf{J}=\frac{1}{m} \operatorname{Im}\left[\psi^{*}(\nabla-i e \mathbf{A}) \psi\right]
$$

The angle $\phi$ which the current makes with the $x$-axis is then given by

$$
\tan \phi=-\frac{J_{y}}{J_{x}} .
$$

Using Eqs. (5) and (9) we can compute the angle of both the incident and the transmitted wave. We find that

$$
\begin{aligned}
\tan \left(\phi_{\text {inc }}\right) & =\frac{k_{y}}{k_{x}} \\
\tan \left(\phi_{\text {trans }}\right) & =\frac{k_{y}+e B d}{\sqrt{k_{x}^{2}-e^{2} B^{2} d^{2}-2 k_{y} e B d}}
\end{aligned}
$$

This agrees exactly with the result obtained for the scattering angle in a purely classical treatment. In fact the result simplifies significantly when $|e B| d<<k_{x}$ in which case

$$
\tan \left(\phi_{\text {trans }}-\phi_{\text {inc }}\right) \simeq \frac{e B d}{k_{x}}
$$

From Eq. (16) we can understand the physical origin of the restriction which was imposed above for $k_{x}^{2}$. When $k_{x}^{2}=e^{2} B^{2} d^{2}+2 k_{y} e B d$ the angle of the transmitted wave $\phi_{\text {trans }}= \pm \pi / 2$ so that for $k_{x}^{2}<e^{2} B^{2} d^{2}+2 k_{y} e B d$ the particle "turns around" and is re-emitted on the right.

It is evident from the above discussion that in order to find $\phi_{\text {trans }}$ given $k_{x}$ and $k_{y}$ it is not necessary to know the detailed form of the wave function in the interaction region. In fact all we actually used to get the scattering angle in the above analysis was our knowledge of the vector potential on both sides of the barrier and the fact that $k_{y}$ was conserved. We shall come back to this point later.

We now proceed to a computation of the reflection and transmission amplitudes $\rho$ and $\tau$. In general exact values can only be obtained numerically since the expressions will necessarily contain the (generally infinite) series $G_{0}(\mathrm{X})$ and $G_{1}(\mathrm{X})$ given in Eqs. (11) and (12) and their derivatives. It is however possible to obtain approximate analytic expressions for $\rho$ and $\tau$ when the magnetic field is small by first relating $G_{0}(\mathrm{X})$ and $G_{1}(\mathrm{X})$ to the confluent hypergeometric functions as follows:

$$
\begin{aligned}
& G_{0}(\mathrm{X})=M\left(-\frac{\lambda}{2}, \frac{1}{2}, \mathrm{X}^{2}\right), \\
& G_{1}(\mathrm{X})=\mathrm{X} M\left(\frac{1}{2}-\frac{\lambda}{2}, \frac{3}{2}, \mathrm{X}^{2}\right),
\end{aligned}
$$

where

$$
\begin{aligned}
M(a, b, z) & =1+\frac{a z}{b}+\frac{(a)_{2} z^{2}}{(b)_{2} 2 !}+\cdots+\frac{(a)_{n} z^{n}}{(b)_{n} n !}+\cdots, \\
(a)_{n} & =a(a+1)(a+2) \cdots(a+n-1) \\
(a)_{0} & =1
\end{aligned}
$$


The confluent hypergeometric functions are in turn related to the Bessel functions by

$$
M(a, b, z)=\Gamma(b) e^{\frac{1}{2} z}\left(\frac{1}{2} b z-a z\right)^{\frac{1}{2}-\frac{1}{2} b} \sum_{n=0}^{\infty} R_{n}\left(\frac{z}{2}\right)^{\frac{n}{2}}(b-2 a)^{-\frac{n}{2}} J_{b-1+n}\left((2 z b-4 z a)^{\frac{1}{2}}\right),
$$

where $R_{0}=1, R_{1}=0, R_{2}=b / 2$, and

$$
R_{n+1}=\left[(n+b-1) R_{n-1}+(2 a-b) R_{n-2}\right] /(n+1),
$$

for $n>2$ [13].

We shall compute $\rho$ and $\tau$ for small $e B d^{2}$ only in the case $k_{y}=0$. To do this we shall need the values of $G_{0}(\mathrm{X}), G_{1}(\mathrm{X})$ and their derivatives at both $x=0$ and $x=d$. When $x=d$, $\mathrm{X}=0$, so these values are easily found by direct substitution into (11) and (12) and the derivatives of these sums. When $x=0$, however, $X=-\sqrt{|e B|} d$, so we use (18), (19), and (23) to obtain expansions for $G_{0}, G_{1}$ and their derivatives in powers of the quantity $\sqrt{|e B|} d$. The calculations are considerably simplified by the fact that " $b$ " in (23) is always a half integer so that the various Bessel functions reduce to simple trigonometric expressions. Furthermore the arguments of the Bessel functions are simply equal to " $k d "$. After a rather lengthy calculation we find, for $|e B| d^{2}<<1$ and $k>>|e B| d$

$$
\begin{aligned}
& \rho=\frac{e^{2} B^{2} d^{4}}{4}\left(\frac{\sin ^{2} k d}{(k d)^{4}}+i\left(\frac{\sin 2 k d}{2(k d)^{4}}-\frac{1}{(k d)^{3}}\right)\right)+O\left(e^{3} B^{3} d^{6}\right) \\
& \tau=1+\frac{e^{2} B^{2} d^{4}}{4}\left(\frac{1}{(k d)^{2}}-\frac{2}{3} \frac{i}{k d}\right)+O\left(e^{3} B^{3} d^{6}\right)
\end{aligned}
$$

Note that the above result satisfies the conservation of flux

$$
|\rho|^{2}+\frac{\tilde{k}}{k}|\tau|^{2}=1
$$

to the required order, $\mathrm{O}\left(e^{2} B^{2} d^{4}\right)$.

\section{SCATTERING FROM AN INFINITE STRIP OF FLUX TUBES}

\section{A. Analytic Results}

In this section we consider the scattering of a charged particle from a regular array of infinitesimally small flux tubes. Our goal is to establish conditions under which this scattering is equivalent to the scattering from a uniform magnetic field whose magnitude is the mean field of all the flux tubes in the array.

To this end consider a situation in which we have an array of infinitesimally small flux tubes in two spatial dimensions each with a flux $\Phi$. We take the lattice to be infinite in extent in the $y$ direction with the flux tubes separated by a distance $\xi$, but to have $N+1$ "columns" in the $x$ direction with flux tubes positioned at $x=0, \xi, \ldots, N \xi$. We shall often choose to have only one column of flux tubes $(N=0)$. (The fact that we have set the spatial separation of the flux tubes in the $x$ and in the $y$ directions to be equal is simply a 
convenience. Our results are easily generalizable to the case of unequal spacing.) We choose the gauge potential of each individual flux tube in a radial gauge relative to the location of the flux tube so that

$$
\begin{gathered}
A_{r}=0 \\
A_{\theta}=\frac{\Phi}{2 \pi r}=-\frac{\zeta}{e r}
\end{gathered}
$$

where $r$ and $\theta$ are the coordinates relative to the position of the flux tube and $\zeta$ provides a convenient parameterization of the flux.

The contributions from each of the flux tubes in the lattice can be summed up to obtain a closed analytic result for the full gauge potential. To do this one begins with the result 14]

$$
\cot \pi z=\frac{1}{\pi} \sum_{n=-\infty}^{\infty} \frac{1}{n+z}
$$

for complex $z$. From this one can derive the formulae

$$
\sum_{m=-\infty}^{\infty} \frac{1}{(m \alpha-\beta)^{2}+\gamma^{2}}=\frac{i \pi}{2 \alpha \gamma}\left[\cot \pi\left(\frac{\beta+i \gamma}{\alpha}\right)-\cot \pi\left(\frac{\beta-i \gamma}{\alpha}\right)\right]
$$

and

$$
\sum_{m=-\infty}^{\infty} \frac{m \alpha-\beta}{(m \alpha-\beta)^{2}+\gamma^{2}}=-\frac{\pi}{2 \alpha}\left[\cot \pi\left(\frac{\beta+i \gamma}{\alpha}\right)+\cot \pi\left(\frac{\beta-i \gamma}{\alpha}\right)\right]
$$

where $\alpha, \beta$ and $\gamma$ are real. Applying these results to our problem gives

$$
\begin{aligned}
& A_{x}=\frac{\zeta \pi}{e \xi} \sum_{n=0}^{N} \frac{\sin \left(\frac{2 \pi y}{\xi}\right)}{\cosh \left(\frac{2 \pi}{\xi}(x-n \xi)\right)-\cos \left(\frac{2 \pi y}{\xi}\right)} \\
& A_{y}=-\frac{\zeta \pi}{e \xi} \sum_{n=0}^{N} \frac{\sinh \left(\frac{2 \pi}{\xi}(x-n \xi)\right)}{\cosh \left(\frac{2 \pi}{\xi}(x-n \xi)\right)-\cos \left(\frac{2 \pi y}{\xi}\right)}
\end{aligned}
$$

where the sum is over the $N+1$ columns of the flux tube array. In fact when $N=0$ the sum collapses to a single term.

Asymptotically (for large positive and negative $x$ ) the gauge potential becomes

$$
\begin{aligned}
& A_{x} \rightarrow 0 \quad \text { as } \quad x \rightarrow \pm \infty \\
& A_{y} \rightarrow \mp \frac{\zeta \pi}{e \xi}(N+1) \quad \text { as } \quad x \rightarrow \pm \infty
\end{aligned}
$$

This asymptotic value is actually reached quite quickly. In fact the first order corrections damp exponentially as $\exp (-2 \pi|x| / \xi)$. Not surprisingly the asymptotic value of the field is precisely that of the constant magnetic field $B$ given in the previous section (Eq. (1)) with 


$$
B=\frac{-2 \zeta \pi}{e \xi^{2}}
$$

and

$$
d=(N+1) \xi
$$

so that $B$ can be interpreted as the mean field of the flux tubes.

One of our goals in this section is to compute the angle by which a charged particle incident from $x=+\infty$ is scattered by the array of flux tubes and to compare the result with the scattering angle off a strip of constant magnetic field. We thus wish to compute the transmitted angle $\phi_{\text {trans }}$ given $k_{x}$ and $k_{y}$ which can be defined asymptotically as in the previous section. In the case of a strip of constant magnetic field we found (in the previous section) that in order to compute the scattering angle it was possible to ignore the behaviour of the vector potential and the wave function in the region $0 \leq x \leq d$ and to simply use the asymptotic forms of these quantities and the fact that $k_{y}$ was conserved. It is tempting to suggest that the same result should apply in the present situation, in which case the scattering angle for the array of flux tubes would be the same as that for the strip of constant field. This result is however only true under certain restricted conditions. The technical problem with the argument is that in our case, due to the lack of (continuous) translational invariance in the $y$ direction, the Schrödinger equation does not separate into $x$ and $y$ pieces. The value of $k_{y}$ is thus not conserved through the "interaction" region of the potential. The discrete translational invariance which is present in our case does, however, lead to interesting predictions. This will be discussed below.

The simplest way to see that the above argument would lead to false conclusions is to consider the case when the flux in each flux tube is an integer. In this case the corrections to the "Mean Field" result are large since the beam is in fact totally undeflected. This follows from the fact that the interaction can be completely "gauged away" by a nonsingular (except possibly at the centres of the flux tubes) single-valued gauge transformation. In fact when $\zeta$ is an integer the wave function

$$
\psi=e^{i\left(-k_{x} x+k_{y} y\right)} \exp \left[i \zeta \sum_{n=0}^{N} \tan ^{-1}\left(\frac{e^{\frac{2 \pi}{\xi}(x-n \xi)}-\cos \left(\frac{2 \pi y}{\xi}\right)}{\sin \left(\frac{2 \pi y}{\xi}\right)}\right)-\frac{i \zeta \pi y}{\xi}\right]
$$

is single-valued and solves Eq. (2) with $\mathbf{A}$ given by Eqs. (33) and (34). The resulting current has $\tan \phi=k_{y} / k_{x}$ everywhere (except possibly at the singularities). In cases where $\zeta$ is not an integer the wave function (38) is multi-valued and is therefore not an admissible solution to the Schrödinger equation. These results were, of course, already noted in the original paper of Aharonov and Bohm 1 . It is thus clear that replacing the flux tubes by a mean field will in general give an incorrect answer. We shall study below the circumstances under which the mean field result is correct.

As one might expect from the fact that there is no scattering when $\zeta$ is an integer, the current (and hence the deflection angle) is a periodic function of $\zeta$. To see this, suppose $\psi_{\zeta}$ is an exact solution to the Schrödinger equation with $0<\zeta<1$. Let $p$ be an integer and perform a gauge transformation with gauge function

$$
\chi=\frac{p}{e} \sum_{n=0}^{N} \tan ^{-1}\left(\frac{e^{\frac{2 \pi}{\xi}(x-n \xi)}-\cos \left(\frac{2 \pi y}{\xi}\right)}{\sin \left(\frac{2 \pi y}{\xi}\right)}\right)-\frac{p \pi y}{e \xi},
$$


This now gives an admissible solution for the case $\zeta \rightarrow p+\zeta$. The current is of course unchanged by the gauge transformation. Thus the scattering angle is periodic in $\zeta$ with a unit period.

It turns out that for certain values of $k_{x}, k_{y}$, and $\zeta$ we can solve for the scattering angle exactly without knowing the form of the wavefunction in the scattering region [15]. The solution depends on the periodicity of the lattice in the $y$ direction [16]. Following the usual discussion of Bloch's theorem [17], one can define a unitary translation operator $T_{n}$ as follows:

$$
T_{n} f(x, y)=f(x, y+n \xi) .
$$

Since our Hamiltonian (with the vector potential defined in (33) and (34) ) is invariant under $y \rightarrow y+n \xi$, we have the operator identity

$$
\left[T_{n}, H\right]=0
$$

Furthermore, the $T_{n}$ 's form a representation of the group of translations by $\xi$ so that

$$
T_{n} T_{m}=T_{m} T_{n}=T_{n+m}
$$

which means that eigenstates of $H$ may be chosen to be simultaneous eigenstates of all of the $T_{n}$ :

$$
\begin{aligned}
H \psi & =E \psi, \\
T_{n} \psi & =c_{n} \psi,
\end{aligned}
$$

with $c_{n} c_{m}=c_{n+m}$. Thus $c_{n}=\exp (2 \pi i n \delta)$ for some real parameter $\delta$, which will be related to $k_{y}$ in this case.

We now consider the form of the wavefunction as $x \rightarrow \infty$ (the incoming wave). In this region we may take (see Eq. (9) )

$$
\psi(x, y)=e^{i\left(k_{y}-\zeta \pi(N+1) / \xi\right) y}\left(e^{-i k_{x} x}+\rho e^{i k_{x} x}\right), \quad x \rightarrow \infty
$$

so that

$$
\delta=\frac{k_{y} \xi}{2 \pi}-\frac{\zeta(N+1)}{2}
$$

To the far left of the scattering region we again seek a plane wave solution, so we set

$$
\psi(x, y)=\tau e^{i\left(k_{y}^{\prime}-\zeta \pi(N+1) / \xi\right) y} e^{-i \tilde{k}_{x} x}, \quad x \rightarrow-\infty .
$$

The fact that $c_{n}=\exp (2 \pi i n \delta)$ then gives

$$
k_{y}^{\prime}=k_{y}+\frac{2 \pi m}{\xi}
$$

Energy conservation then determines $\tilde{k}_{x}$ : 


$$
\tilde{k}_{x}^{m}=\left[k_{x}^{2}-\left(\frac{2 \pi}{\xi}\right)^{2}(\zeta(N+1)-m)^{2}+\frac{4 \pi k_{y}}{\xi}(\zeta(N+1)-m)\right]^{\frac{1}{2}},
$$

so that if $\tilde{k}_{x}^{m}$ is real the angle of the beam of transmitted particles is determined by the equation

$$
\tan \phi_{\operatorname{trans}}^{m}=\frac{k_{y}-\frac{2 \pi}{\xi}(\zeta(N+1)-m)}{\left[k_{x}^{2}-\left(\frac{2 \pi}{\xi}\right)^{2}(\zeta(N+1)-m)^{2}+\frac{4 \pi k_{y}}{\xi}(\zeta(N+1)-m)\right]^{\frac{1}{2}}}
$$

Comparing the above equation with Eqs. (16), (36) and (37), we see that agreement with the MF result is achieved when $m=0$.

We now restrict ourselves to the case where $N=0$ and consider plotting tan $\phi_{\text {trans }}$ versus $\zeta$. The "critical points" (i.e., the points for which $\tilde{k}_{x}^{m}=0$ ) are given by

$$
\zeta_{m}^{ \pm}=m+\frac{k_{y} \xi}{2 \pi} \pm\left(k_{x}^{2}+k_{y}^{2}\right)^{\frac{1}{2}} \frac{\xi}{2 \pi} .
$$

If $\left(k_{x}^{2}+k_{y}^{2}\right)^{\frac{1}{2}} \xi \leq \pi$, the scattering angle is well defined in the intervals $\left(\zeta_{m}^{-}, \zeta_{m}^{+}\right)$and is given by Eq. (50). In the intervals $\left(\zeta_{m}^{+}, \zeta_{m+1}^{-}\right)$, all the $\tilde{k}_{x}^{m}$ are imaginary and the beam of particles is backscattered by the flux tubes. If $\left(k_{x}^{2}+k_{y}^{2}\right)^{\frac{1}{2}} \xi>\pi$, there are regions of the $\zeta$ axis for which more than one scattering angle could be defined. Had the incident beam been characterized by a wave packet in our calculations, the scattered particles would have split (asymptotically) into several wave packets with distinct directions in space, given by (50). Since our incident beam is characterized by a plane wave the scattered wave can never be separated into its various pieces and the scattering angle is not well defined. If we view the situation from the perspective of diffraction theory, we see that the "circular waves" being emitted from each of the scattering centers are interfering constructively in more than one direction.

A similar result holds for any value of $N$. There may be certain values of the parameters for which there is no value of $m$ with real $\tilde{k}_{x}^{m}$. In such cases the beam of particles is totally reflected by the flux tubes. If there is only one integer $m$ for which $\tilde{k}_{x}^{m}$ is real the scattering angle is well defined and is given by (50). If, in addition, $m=0$, the angle agrees exactly with the MF case. Finally, it is possible that there exist more than one integer $m$ for which $\tilde{k}_{x}^{m}$ is real. This situation corresponds to the "diffraction" case which was considered above.

Finally, note that the invariance of $\phi_{\text {trans }}$ under $\zeta \rightarrow \zeta+m^{\prime}$ (which is expected in light of the gauge invariance arguments given above) is already included in Eq. (50) by simply taking $m \rightarrow m-m^{\prime}(N+1)$.

\section{B. Numerical Results}

The above analytic analysis is somewhat limited in that it only predicts the scattering angles and in that it depends in an essential way on the periodicity of the lattice. We shall now develop a numerical technique which will allow us to examine not only the scattering angle but also the lowest order values of the reflection and transmission amplitudes. This technique can be generalized to a non-periodic array of flux tubes.

As discussed in the introduction, we expect the Mean Field result to be valid when the flux per "particle" $\zeta$ is small. We thus analyze our problem for small $\zeta$. Consider a wave 
which is incident from $x=+\infty$. This incident wave will induce a scattered wave from each of the flux tubes in the lattice. To linear order in $\zeta$ the total wave function can be viewed as the incident wave plus the sum of the scattered waves from each individual flux tube. This procedure is, however, significantly more complicated than in ordinary scattering theory due to the presence of the Aharonov-Bohm phases.

In order to proceed we shall begin by reviewing the results for scattering from a single flux tube which we assume is positioned at the origin. In this case, an exact scattering solution is known [4]. It is given by

$$
\psi=\sum_{m=-\infty}^{\infty} e^{-\frac{i \pi}{2}|m+\zeta|} J_{|m+\zeta|}(k r) e^{i m \theta},
$$

where $J$ is a Bessel function and the angle $\theta$ should be taken from $-\pi$ to $\pi$ (where $\theta=0$ corresponds to the positive $x$ axis). To see that this is a scattering solution one notes that as $x \rightarrow \infty$

$$
\psi \simeq e^{-i k x-i \zeta \theta}
$$

so that the incident current density is in the negative $x$ direction. In fact for $|\zeta|<1$, Aharonov and Bohm (with modifications due to Hagen [18]) showed that (52) reduces asymptotically (as $r \rightarrow \infty)$ to

$$
\psi \rightarrow e^{-i k x-i \zeta \theta} \mp i \frac{e^{i k r}}{(2 \pi i k r)^{1 / 2}} \sin \zeta \pi \frac{e^{\mp i \theta / 2}}{\cos (\theta / 2)},
$$

where the upper (lower) sign holds for $\zeta$ positive (negative). The above asymptotic expression for $\psi$ requires one further crucial assumption which is that $(k r(1 \pm \cos \theta))^{1 / 2}$ are large. This assumption fails in the forward and backward directions so that (54) is inaccurate in these regions. This is fortunate since (52) is convergent and single-valued for all $\theta$ as can be confirmed by a direct evaluation of (52) near $\theta= \pm \pi$. The asymptotic form (54), however, is not single valued and it diverges when $\theta= \pm \pi$.

This "splitting" of the wave, in the case of a single scatterer, into an incident and a scattered wave is essential for us since it is the starting point for summing up the scattered waves from a lattice of flux tubes. We cannot simply use Eq. (54) for this splitting, even though the "scattered" wave is proportional to $\sin (\zeta \pi)$ which is small for small $\zeta$, since Eq. (54) is not valid for all $\theta$. Eq. (54) does however suggest a more appropriate way to define the scattered wave. We consider the definition:

$$
\psi_{\text {scat }}=\psi_{\text {full }}-e^{-i k x-i \zeta \theta}
$$

where $\psi_{\text {full }}$ is the full wave function given in Eq. (52) which can be evaluated (for example) numerically. This definition is not without problems since, of course, $\psi_{\text {scat }}$ is not singlevalued. There are however several ways to circumvent this problem and to obtain useful results with this approach.

The most aesthetically pleasing approach to dealing with this "multi-valuedness" problem is to work with the currents rather than with the wave functions whenever possible. We begin by taking the covariant derivative of (52) directly. This gives the simple result 


$$
(\nabla-i e \mathbf{A}) \psi=-\mathbf{i} i k \psi+\mathbf{i} f_{x}+\mathbf{j} f_{y},
$$

where

$$
\begin{aligned}
& f_{x}=\frac{k}{2}\left[e^{i \zeta \pi / 2}\left(e^{-i \theta} J_{-\zeta+1}-i J_{-\zeta}\right)+e^{-i \zeta \pi / 2}\left(e^{-i \theta} J_{\zeta-1}+i J_{\zeta}\right)\right], \\
& f_{y}=\frac{k}{2}\left[e^{i \zeta \pi / 2}\left(i e^{-i \theta} J_{-\zeta+1}-J_{-\zeta}\right)+e^{-i \zeta \pi / 2}\left(i e^{-i \theta} J_{\zeta-1}+J_{\zeta}\right)\right] .
\end{aligned}
$$

The Schrödinger equation can now be used to derive an equation satisfied by $f_{x}$ and $f_{y}$. Applying the covariant derivative to (56) gives the relation

$$
-i k f_{x}+\frac{\partial f_{x}}{\partial x}+\frac{\partial f_{y}}{\partial y}-i e A_{x} f_{x}-i e A_{y} f_{y}=0,
$$

which may be verified explicitly.

For the case of a lattice of flux tubes the covariant derivative of the total wave function may be obtained, to leading order in $\zeta$, by adding, to the term $-\mathbf{i} i k \psi_{\text {total }}$, the sum of the contributions of the $f_{x}$ 's and $f_{y}$ 's from each flux tube. We shall specialize to the case when there is only one column of flux tubes and when $k_{y}=0$ (i.e. normal incidence). We shall discuss the more general case later. Let us define the vector $\Omega(x, y)$ as the sum of the contributions of $f_{x}$ and $f_{y}$ from all the flux tubes in the lattice. That is

$$
\begin{aligned}
& \Omega_{x}(x, y)=\sum_{n=-\infty}^{\infty} f_{x}^{n}\left(k r_{n}, \theta_{n}, \zeta\right), \\
& \Omega_{y}(x, y)=\sum_{n=-\infty}^{\infty} f_{y}^{n}\left(k r_{n}, \theta_{n}, \zeta\right),
\end{aligned}
$$

where

$$
r_{n}=\left(x^{2}+(y-n \xi)^{2}\right)^{1 / 2},
$$

and where $\theta_{n}=\pi$ is parallel to the $x$-axis and corresponds to the direction of propagation of the incident wave. To lowest order in $\zeta$ the total wave function satisfies

$$
(\nabla-i e \mathbf{A}) \Psi=(-\mathbf{i} i k) \Psi+\Omega
$$

This is analagous to what is done in ordinary scattering theory where the scattered waves from each individual atom are summed to obtain the total scattered wave.

To further check the self consistency of the above procedure we apply the covariant derivative to Eq. (62). $\Psi$ is expected to satisfy the Schrödinger equation far away from any of the flux tubes. (In fact in our case it will turn our that "far away" may be as close as a few lattice spacings.) It will do so provided $\boldsymbol{\Omega}$ satisfies the equation

$$
-i k \Omega_{x}+\frac{\partial \Omega_{x}}{\partial x}+\frac{\partial \Omega_{y}}{\partial y}-i e A_{x} \Omega_{x}-i e A_{y} \Omega_{y}=0
$$

where $A_{x}$ and $A_{y}$ are the total gauge potentials given in (33) and (34). Consider the asymptotic region on the left where Eq. (35) holds. Applying (59) to every term in the sums in (63) gives 


$$
i \zeta \pi\left[\sum_{n}\left(\frac{y}{\pi r_{n}^{2}} f_{x}^{n}-\frac{x}{\pi r_{n}^{2}} f_{y}^{n}\right)-\frac{\Omega_{y}}{\xi}\right]=O\left(\zeta^{2} \pi^{2}\right) .
$$

It is easy to see that the $f$ 's are, asymptotically, of order $\zeta \pi$. Thus the Schrödinger equation will be satisfied asymptotically provided $\Omega$ is also of order $\zeta \pi$. Eq. (60) guarantees, that this will be the case.

We have not been able to obtain an analytic expression for $\Omega$, even asymptotically. We have thus chosen to evaluate $\Omega$ numerically. Some details of the numerical work are discussed in the Appendix. We have found that to a very high precision and for a range of $k$ which is also discussed in the Appendix (basically $2 \pi>k \xi>>2 \zeta \pi$ )

$$
\boldsymbol{\Omega} \simeq-\frac{2 i}{\xi} \sin (\zeta \pi) e^{-i k x} \mathbf{j}
$$

This result is valid provided one is not too close to the array of flux tubes. Note also that to this order in $\zeta$ the value of $k$ (which is actually $k_{x}$ ) is the same for the incident and the transmitted wave. Also, as expected, when $\zeta \pi$ is small (64) is satisfied.

The next step in evaluating the scattering angle is to compute the current. Using (62) the current is found to be

$$
\begin{aligned}
\mathbf{J} & =\frac{1}{m} \operatorname{Im}\left[\Psi^{*}(\nabla-i e \mathbf{A}) \Psi\right], \\
& =\frac{1}{m}\left[-\mathbf{i} k|\Psi|^{2}+\operatorname{Im}\left(\Psi^{*} \Omega\right)\right]
\end{aligned}
$$

This expression could potentially cause us some difficulty since it involves $\Psi$ directly whereas we have only computed the covariant derivative of $\Psi$. Fortunately, to the order in $\zeta$ to which we are working, there is no ambiguity in $\Psi$ and we can take $\Psi$ to be simply the incident wave since linear order corrections will lead to quadratic order corrections to the scattering angle. Thus

$$
\Psi=e^{-i k x}+O(\zeta \pi)
$$

and

$$
|\Psi|^{2}=1+O(\zeta \pi)
$$

We are now ready to use Eq. (67) to evaluate the scattering angle $\phi_{\text {trans }}$.

$$
\tan \phi_{\text {trans }}=-\frac{2 \zeta \pi}{k \xi}+O\left(\zeta^{2} \pi^{2}\right)
$$

Eqs. (17) and (36) can now be used to compare this result with the scattering off of a strip of constant magnetic field. We see that to lowest order in $\zeta \pi$ the scattering from an array of flux tubes agrees exactly with the Mean Field result (which coincides with the analytic result Eq. (50) in this case). This agreement provides a good check of our numerical method.

In the previous discussion we have avoided summing the non single-valued scattered wave functions by summing only their covariant derivatives. It is however possible to sum the scattered waves directly without first evaluating $D \psi$. Using the definition (55) of the 
scattered wave from an individual flux tube we note that the scattered wave at $(x, y)$ due to a flux tube located at $n \xi$ is given by

$$
\psi_{\text {scat }}^{n}\left(k r_{n}, \theta_{n}, \zeta\right)=\left(\psi\left(k r_{n}, \theta_{n}, \zeta\right)-e^{-i k r_{n} \cos \theta_{n}-i \zeta \theta_{n}}\right)
$$

where $\psi$ is the full wave function for each individual scatterer and $r_{n}$ and $\theta_{n}$ are defined as in the previous discussion. To lowest order in $\zeta$ the total scattered wave will be the sum of the waves scattered from each individual flux tube:

$$
\Psi_{\text {scat }}=\sum_{n=-\infty}^{\infty} \psi_{\text {scat }}^{n}\left(k r_{n}, \theta_{n}, \zeta\right) \text {. }
$$

We know of no way to do this sum analytically. We have thus evaluated (72) numerically by summing the terms until satisfactory convergence was achieved. For $k r_{n}$ large and $\theta_{n}$ near $\pm \pi / 2$ the asymptotic form (54) for $\psi$ for each flux tube was used. In other cases the sum (52) was evaluated directly. This sum converges fairly rapidly for small and moderate values of $k r_{n}$ since for large index

$$
J_{n}(x) \sim \frac{1}{\sqrt{2 \pi n}}\left(\frac{e x}{2 n}\right)^{n} .
$$

The numerical work showed that to the left of the scattering region and for momenta in the range $2 \pi>k \xi>>2 \zeta \pi$ the scattered wave has the approximate form

$$
\Psi_{\mathrm{scat}} \simeq e^{-i k x}(\epsilon(x, y)+i \sin (\zeta \pi) \chi(y)) .
$$

For $k \xi$ not too small $\epsilon$ was found to be negligible compared to $\zeta \pi$ and $\chi$ was found to have the approximate form:

$$
\chi(y) \simeq-\frac{2(y-n \xi)}{\xi}+\frac{k \xi-1}{k \xi} \quad \text { for } n \xi<y<(n+1) \xi
$$

for $\infty<n<+\infty$. In fact $\chi$ does have some oscillatory $x$ dependence but it was found to be negligible compared to the $y$ dependence of $\chi$. The slope " $-2 / \xi$ " is accurate to about $.2 \%$, and $(k \xi-1) / k \xi$ is accurate to about $1 \%$. For a more thorough discussion of $\epsilon$ and $\chi$ and of the limits on the momentum we again refer the reader to the Appendix.

It is clear from our method that $\Psi_{\text {scat }}$ must have discontinuities along lines starting at the site of each flux tube and extending out to the left parallel to the $x$ axis. This is because we have subtracted out the multi-valued incident wave $\exp (-i k x-i \zeta \theta)$ from each (singlevalued) wave function. This brings up the difficult problem of what to choose as the incident wave in the multi-vortex case. Although it is true that to lowest order it is simply a plane wave we must be very careful since the scattered wave (as we have defined it) is not single valued.

One important condition which $\Psi_{\text {inc }}$ should satisfy is

$$
(\nabla-i e \mathbf{A}) \Psi_{\mathrm{inc}}=-\mathbf{i} i k \Psi_{\mathrm{inc}}
$$

The form $\Psi_{\text {inc }}=\exp (-i k x+i \zeta \pi y / \xi)$ satisfies this condition asymptotically $($ when $x<<0)$. However due to the form of $\chi$ which has discontinuities resulting from its multi-valuedness it is important to choose $\Psi_{\text {inc }}$ to have the same discontinuities. In fact 


$$
\Psi_{\text {inc }}=e^{-i k x}\left(1+i \zeta \pi \frac{(y-n \xi)}{\xi}\right) \quad \text { for } n \xi<y<(n+1) \xi
$$

also satisfies Eq. (76) asymptotically but only to order $\zeta \pi$. Setting

$$
\Psi=\Psi_{\text {inc }}+\Psi_{\text {scat }},
$$

we can compute the covariant derivative of the total wave function $\Psi$. To linear order in $\zeta \pi$

$$
(\nabla-i e \mathbf{A}) \Psi=-\mathbf{i} i k \Psi-\mathbf{j} \frac{2 i}{\xi} \zeta \pi e^{-i k x}
$$

This is precisely the result which we obtained using our previous method (see Eqs. (62) and (65) ). We thus see that with this method as well, we recover the Mean Field result when the flux per particle is small. Note that we have shown that $\Psi$ is a plane wave with corrections linear in $\zeta \pi$. This was assumed in our previous discussion (Eq. (68)).

It likely that the linear correction to the plane wave above should be exponentiated. From this point of view the discontinuities are very useful since they allow the perturbative corrections to $\psi$ to remain small even for large $y$.

It is by now clear that the two approaches discussed above are complementary. In the former approach it appeared as though we could avoid any problems of discontinuities but in fact the discontinuities were all "hidden" in $\Psi$. The latter approach has the advantage of showing clearly what is going on by evaluating the wave function directly. From a calculational point of view the former approach is advantageous (at least in lowest order) since the derivative of $\Psi$ is found directly without having to evaluate $\Psi$ at several points and perform a linear regression to find the slope.

It is a simple matter to extend the above results to the case where $k_{y}$ is nonzero. A subtlety which one encounters numerically is that the wave functions (or their covariant derivatives) due to each flux tube must be multiplied by appropriate phases in order that the plane waves incident at each site are in phase. Our two methods again give complementary results so we describe here only the "first" method in which the covariant derivative or $\Psi$ is taken first. The appropriate generalization of (62) is

$$
(\nabla-i e \mathbf{A}) \Psi=\left(-\mathbf{i} i k_{x}+\mathbf{j} i k_{y}\right) \Psi+\Omega,
$$

where $\Omega$ has been studied numerically and is approximately given by

$$
\boldsymbol{\Omega} \simeq-\frac{2 i}{\xi} \sin (\zeta \pi) e^{i\left(k_{y} y-k_{x} x\right)}\left(\frac{2 k_{y}}{k_{x}} \mathbf{i}+\mathbf{j}\right)
$$

in the asymptotic region on the left. We refer the reader to the Appendix for more details of the numerical results. Generalizing from the result for $k_{y}=0$ we take

$$
\Psi=e^{i\left(k_{y} y-k_{x} x\right)}+O(\zeta \pi)
$$

We then find

$$
\tan \left(\phi_{\text {trans }}-\phi_{\text {inc }}\right)=-\frac{2 \zeta \pi}{k_{x} \xi}+O\left(\zeta^{2} \pi^{2}\right)
$$


This result again agrees with the Mean Field result (Eq. (17)) and with the exact result, since " $m "=0$.

The extension of our results to the case where there are several columns is also a simple matter provided the perturbative analysis remains valid. Suppose we were to add a new column positioned at $x=\xi$. In the local coordinates $\left(x^{\prime}, y^{\prime}\right)$ of that column an incident plane wave $\exp \left(i k_{y} y^{\prime}-i k_{x} x^{\prime}\right)$ gives rise to

$$
\mathbf{\Omega}^{\prime}=-\frac{2 i}{\xi} \sin (\zeta \pi) e^{i\left(k_{y} y^{\prime}-k_{x} x^{\prime}\right)}\left(\frac{2 k_{y}}{k_{x}} \mathbf{i}+\mathbf{j}\right) .
$$

Since $y^{\prime}=y$ and $x^{\prime}=x-\xi$ the wave incident on the column at $x=\xi$ must be multiplied by the phase $\exp \left(-i k_{x} \xi\right)$ to obtain the correct phase of the plane wave at the locations of the two columns. Thus

$$
\boldsymbol{\Omega}_{\mathrm{tot}}=\boldsymbol{\Omega}+e^{-i k_{x} \xi} \mathbf{\Omega}^{\prime}=2 \boldsymbol{\Omega}
$$

so that

$$
\tan \left(\phi_{\text {trans }}-\phi_{\text {inc }}\right)=-\frac{4 \zeta \pi}{k_{x} \xi}+O\left(\zeta^{2} \pi^{2}\right)
$$

for $N=1$. In general, for $N+1$ columns,

$$
\tan \left(\phi_{\text {trans }}-\phi_{\text {inc }}\right)=-\frac{2(N+1) \zeta \pi}{k_{x} \xi}+O\left(\zeta^{2} \pi^{2}\right)
$$

The net effect is that our perturbative expansion is now in " $(N+1) \zeta \pi$ " rather than in " $\zeta \pi$ ". Thus even for multiple columns the scattering angle agrees with the exact result and thus with that obtained from the Mean Field analysis provided the flux per particle is sufficiently small. Again this agreement provides a good check of our numerical method.

So far we have concentrated on comparing the scattering angle for the lattice of flux tubes with that obtained in the Mean Field analysis. We now describe a comparison of another quantity of interest namely the transmission and reflection amplitudes $\tau$ and $\rho$, for which there are no exact results available. We shall discuss only the case $k_{y}=0$ for which we shall compute numerically the sum in Eq. (72).

In the previous section we computed $\rho$ and $\tau$ for the Mean Field case. In terms of the "flux parameters" $\zeta$ and $\xi$ and in the limit when $\zeta \pi$ is small and $k \xi>>|\zeta| \pi$ they are given by (see Eqs (36), (37), (25) and (26)):

$$
\begin{aligned}
& \rho_{\mathrm{mf}}=\mathrm{e}^{i k \xi} \zeta^{2} \pi^{2}\left(\frac{\sin ^{2} k \xi}{(k \xi)^{4}}+i\left(\frac{\sin 2 k \xi}{2(k \xi)^{4}}-\frac{1}{(k \xi)^{3}}\right)\right)+O\left(\zeta^{3} \pi^{3}\right) \\
& \tau_{\mathrm{mf}}=1+\zeta^{2} \pi^{2}\left(\frac{1}{(k \xi)^{2}}-\frac{2}{3} \frac{i}{k \xi}\right)+O\left(\zeta^{3} \pi^{3}\right) .
\end{aligned}
$$

(The extra factor of $\mathrm{e}^{i k \xi}$ has been added to compensate for the positioning of the mean field relative to the flux tubes.) Note that to order $\zeta \pi$ we have the simple result that $\rho=0$ and $\tau=1$. To this order the wave is entirely transmitted in agreement with the classical result. We now describe the calculation for $\rho$ and $\tau$ for the lattice of flux tubes. 
We begin by summing (72) numerically. When $k \xi>>2|\zeta| \pi$ and $x>0$ we find

$$
\Psi_{\text {refl }}=-\frac{i}{k \xi} \sin \zeta \pi e^{i k x},
$$

to an accuracy of about $1 \%$ in the coefficient of " $-i / k \xi "$. A naive interpretation of this result would imply

$$
\rho_{\mathrm{ft}}=-\frac{i \zeta \pi}{k \xi}+O\left(\zeta^{2} \pi^{2}\right)
$$

which is linear in $\zeta \pi$. This, of course, differs from the Mean Field result. We now argue that this naive interpretation is incorrect and that to order $\zeta \pi$ we must be more careful in defining the reflection and transmission amplitudes. Physically this is due to the fact that the angle of the wave has been changed to order $\zeta \pi$ by the scattering. This change in angle should not affect the transmission amplitude. More concretely recall that to the left of the scattering region the scattered wave has the form

$$
\Psi_{\text {scat }}=i \sin (\zeta \pi) \chi(y) e^{-i k x}
$$

( $\epsilon$ is negligible for large $k \xi$ ) and that $\chi(y)$ is a "sawtooth" with discontinuities whenever $y=n \xi$. We have discussed previously that $\chi$ must somehow exponentiate to give the correct plane wave in the $y$ direction. It is thus incorrect to read off the transmission coefficient naively from Eq. (92) which would give

$$
\tau_{\mathrm{ft}}=1+i \zeta \pi \bar{\chi}+O\left(\zeta^{2} \pi^{2}\right)
$$

In fact the contribution of $\zeta$ is simply to modify the plane wave to take into account the "bending" of the particle in the magnetic field and the correct $\tau$ to this order is one. Thus the apparent $O(\zeta \pi)$ correction to the transmission amplitude is best understood as an artifact of our approach and the difficulties which the Aharonov-Bohm phases cause when one tries to add up the scattered waves.

Once we realize that $\tau$ is one to order $\zeta \pi$ it is clear that $\rho$ must vanish to this order. This follows from flux conservation. The fact that the $\rho_{\mathrm{ft}}$ which we naively calculated is of order $\zeta \pi$ is a direct consequence of the fact that the "naive" $\tau_{\mathrm{ft}}$ has a piece which is linear in $\zeta \pi$ since

$$
\left|\rho_{\mathrm{ft}}\right|^{2}+\frac{\tilde{k}}{k}\left|\tau_{\mathrm{ft}}\right|^{2} \simeq \frac{\zeta^{2} \pi^{2}}{k^{2} \xi^{2}}+\left(1-\frac{2 \zeta^{2} \pi^{2}}{k^{2} \xi^{2}}\right)\left(1+\frac{\zeta^{2} \pi^{2}}{k^{2} \xi^{2}}\right) \simeq 1
$$

where we have used the fact (from Eq. (75)) that

$$
\chi \overline{(y)} \equiv \chi(y=.5 \xi) \simeq-\frac{1}{k \xi},
$$

It may of course happen that $\tau_{\mathrm{ft}}$ has real pieces quadratic in $\zeta \pi$ (to which our calculation would be insensitive). In this case these should be included in $\left|\tau_{\mathrm{ft}}\right|^{2}$. In fact the above argument used numerical results and it is thus just valid in an approximate sense. In any case it is evident that the apparent discrepancy between the form of $\rho$ and $\tau$ in the "flux tube" and the Mean Field case is simply an artifact of our approach. 


\section{DISCUSSION}

In this paper we set out to understand the validity of Mean Field Theory when applied to scattering from infinitesimally small flux tubes for which there is no Lorentz force except possibly at the location of the flux tubes. We considered the scattering of a plane wave representing an incident charged particle from an array of flux tubes with infinite extent in the $y$ direction. By computing the current of the wave beyond the scattering region we found that the incident beam was bent by the array of flux tubes. When the flux per tube is small the angle by which the beam is bent is given precisely by the Mean Field result.

Caenepeel and MacKenzie [8] have recently shown that no bending occurs when scattering from a single flux tube unless an additional interaction besides the Aharonov-Bohm interaction is present [19]. From our calculation one can see that even in the absense of an additional interaction there is precisely the correct (MF) amount of bending from an array of flux tubes. Although our calculation was done (for essential technical reasons) for a regular lattice, it seems very likely on physical grounds that the main results will persist for a random array of flux tubes.

Despite the absense of a Lorentz force for a lattice of flux tubes there is a way to understand how such bending can occur classically. Imagine first taking each flux tube to be of finite but small size, computing the scattering, and then letting the size become very small keeping the mean field, and thus the flux per tube, constant. One finds that the charged particle will have to travel an increasingly large distance before scattering but, after many interactions, the mean impulse it receives from all the tubes from which it scatters is precisely that which it would have received from the mean field. There is however a catch. This only works when the flux tubes are larger than some critical size at which the magnetic field inside the flux tube becomes so large that the radius of the path of the particle is equal to the radius of the flux tube. Beyond this critical size, the average deflection which the particle receives becomes too small. It is only Quantum Mechanical scattering to which the Mean Field result truly applies when the flux per tube is small.

\section{APPENDIX: COMMENTS ON THE NUMERICAL WORK}

In this appendix we describe several interesting features of our numerical work which were not mentioned in the text.

We begin by discussing the numerical work leading to Eq. (74) for $\Psi_{\text {scat }}$. Recall that for $k_{y}=0$ we claimed that our numerical results lead to a scattered wave of the form

$$
\Psi_{\mathrm{scat}} \simeq e^{-i k x}(\epsilon+i \sin (\zeta \pi) \chi(y))
$$

with

$$
\chi(y) \simeq-\frac{2(y-n \xi)}{\xi}+\frac{k \xi-1}{k \xi}
$$

in the region to the left of the flux tubes. The result (96) was obtained by summing the

series in Eq. (72) numerically until satisfactory convergence was achieved. For very small $k \xi$ this involved adding the contributions from up to a million flux tubes in order to obtain 
a slope in $\chi$ accurate to about $.2 \%$. As one might expect from the fact that the vector potential approaches the MF result exponentially (c.f. Eqs. (33) - (35) ) the above formula becomes valid within a very short distance of the scattering region (i.e. within a distance of about $4 * \xi)$.

For $k \xi$ very large compared to $\zeta \pi, \epsilon$ was negligible. For $k \xi$ closer to $\zeta \pi$ the maximum value of $|\epsilon|$ was found to be approximately proportional to $\zeta \pi$ and to have an approximate $1 / k \xi$ dependence. From (97) $\chi$ is also dominated by a $1 / k \xi$ dependence for small $k \xi$. For all cases investigated (we examined $\zeta$ as low as .0001 and $k \xi$ as low as .005) it has been found that

$$
|\epsilon|<<\sin (\zeta \pi)|\chi|
$$

for small $k \xi$.

The growth of $\chi$ with small $k \xi$ has an interesting interpretation. From a MF point of view we would expect the particle to be totally reflected for $k \xi<2|\zeta| \pi$. From our perturbative approach we see that the results become non-perturbative when $\zeta \pi \chi$ becomes of order 1 i.e. when $k \xi \sim|\zeta| \pi$. In a loose sense our approach predicts the correct "critical" point.

Numerical work was also required for evaluating $\Omega$ defined in Eq. (60). In order to determine $\Omega$ for the case $k_{y}=0$ the exact forms of $f_{x}$ and $f_{y}$ were used for small $k r$. For larger $k r$ approximate forms (easily derived from (57) and (58)) were employed. As in the previous calculation it was necessary to sum up to a million tubes. For $k \xi$ very large compared to $2 \zeta \pi$ and slightly less than $2 \pi$ the form quoted for $\Omega_{y}$ in (65) was accurate to about $.2 \%$. Similarly, $\Omega_{x} / \Omega_{y}$ was "zero" to a magnitude of about .002 .

For the more general case where $k_{y}$ is nonzero, things are much the same. A small difference was that some precision was lost in $\Omega_{x}$ when $k_{y}<<k_{x}$. The reason is that the number which was expected was proportional to $k_{y} / k_{x}$ and thus became very small.

For large momenta it was found that $\Psi_{\text {scat }}$ and $\Omega$ deviated from the forms quoted in the main body of the paper due to diffraction effects. Recall that diffraction occurs when circular waves "emanating" from neighbouring sites add up coherently. For small momenta there is only one angle at which this occurs and only the "forward peak" is observed. For higher momenta it becomes possible to have coherence in more than one direction. In effect, the transmitted wave becomes the sum of more than one plane wave. As discussed near the end of Section III.A, the scattering angle then becomes ill defined.

One can derive the limit on $k_{x}$ and $k_{y}$ by using Eq.(51) for the "critical" fluxes. Since our numerical approach is only valid near $\zeta=0$, we shall for convenience set $\zeta=0$ for this derivation. Diffraction then starts when either $\zeta_{-1}^{+}$or $\zeta_{+1}^{-}$is equal to zero so that the limit on the momenta is easily seen to be

$$
\left(k_{x}^{2}+k_{y}^{2}\right)^{\frac{1}{2}} \xi+\left|k_{y}\right| \xi<2 \pi .
$$

\section{Acknowledgements}

This work is supported in part by the Natural Sciences and Engineering Research Council of Canada. Their support is gratefully acknowledged. We wish to thank Ian Kogan, Arnold Sikkema and Stephanie Curnoe for helpful discussions. 


\section{REFERENCES}

[1] A comprehensive reference is "The Quantum Hall Effect", R. Prange and S. Girvin, editors, Springer-Verlag (1987).

[2] R. B. Laughlin, Science 242 (1988) 525;

[3] S.C. Zhang, Int. J. Mod. Phys. B6, 25(1992)

[4] Y. Aharonov and D. Bohm, Phys. Rev. 115 , 485, (1959).

[5] See for example J. McCabe and R. MacKenzie, "Mean-Field Theory Near Bose Statistics"; University of Montreal Preprint UdeM-LPN-TH-102; (1992).

[6] V. Kalmeyer and R. B. Laughlin, Phys. Rev. Lett. 59 (1987) 2095; A. L. Fetter, C. B. Hanna and R. B. Laughlin, Phys. Rev. B39 (1989) 9679 and Phys. Rev. B40 (1989) 8745; Y. H. Chen, B. I. Halperin F. Wilczek and E. Witten, Int. J. Mod. Phys. B3 (1989) 1001; J. Lykken, J. Sonnenschein, and N. Weiss, Phys. Rev. D42 (1990) 2161;

[7] Two papers on a related topic are: C. Gros, et al, Phys. Rev.B43, 5883 (1991) and M. D. Johnson, et al, "Vector Mean Field Theory of Statistics," Proceedings of the University of Miami Workshop on: Electronic Structure and Mechanisms for High-Temperature Superconductivity, 1991.

[8] D. Caenepeel and R. MacKenzie, "Parity Violation and the Mean Field Approximation for the Anyon Gas"; University of Montreal preprint UdeM-LPN-TH-130; hepth/9303078; (March 1993).

[9] E. Fermi, Nuclear Physics, compiled by J. Orear et al (The University of Chicago Press, Chicago, 1974), pp. 201-202.

[10] J. D. Jackson, Classical Electrodynamics (John Wiley and Sons, Inc., Toronto, 1975), Second Edition, pp. 454-459.

[11] Z-Y Gu and S-W Qian, J. Phys. A: Math. Gen. 21, 2573 (1988) (Corrigendum: J. Phys. A: Math. Gen. 23, 4183 (1990)). For a comment on the above paper, see: A. D. de Veigy and S. Ouvry, Phys. Lett. B307, 91 (1993).

[12] G. Arfken, Mathematical Methods for Physicists (Academic Press, Inc., Orlando, 1985), Third Edition, p. 465.

[13] M. Abramowitz and I.A. Stegun, Handbook of Mathematical Functions (General Publishing Company, Ltd., Toronto, 1972), Chapter 13.

[14] T. J. I'A. Bromwich,An Introduction to the Theory of Infinite Series(MacMillan and Co. Ltd., London, 1965), Second Edition, p. 296.

[15] We thank the referee for pointing out to us that there are certain conditions under which this method leads to a unique scattering angle. This made it possible for us to include the details of the analytic results in this paper.

[16] Although we treat the problem using Bloch's theorem, it is possible to arrive at the same result via the "modular variables" of Aharonov, et al. See Y. Aharonov, H. Pendelton and A. Peterson, Int. J. of Theor. Phys. 2, 213 (1969).

[17] For a more thorough treatment of Bloch's theorem, see for example, N. W. Ashcroft and N. D. Mermin, Solid State Physics (Harcourt Brace Jovanovich College Publishers, New York, 1976), pp. 133-141.

[18] C. R. Hagen, Phys. Rev. D41, 2015 (1990).

[19] J. March-Russell and F. Wilczek, Phys. Rev. Lett. 61, 2066 (1988). 